\title{
Phase dynamics after connection of two separate Bose-Einstein condensates
}

\author{
I. Zapata, ${ }^{1,2}$, A F. Sols, ${ }^{2}$ and A. J. Leggett ${ }^{1}$ \\ ${ }^{1}$ Department of Physics, University of Illinois at Urbana-Champaign, 1110 West Green Street, Urbana, IL 61801 \\ ${ }^{2}$ Departamento de Física Teórica de la Materia Condensada and Instituto de Ciencia de Materiales "Nicolás Cabrera", \\ Universidad Autónoma de Madrid, E-28049 Madrid, Spain
}

(Dated: November 16, 2018)

\begin{abstract}
We study the dynamics of the relative phase following the connection of two independently formed Bose-Einstein condensates. Dissipation is assumed to be due to the creation of quasiparticles induced by a fluctuating condensate particle number. The coherence between different values of the phase, which is characteristic of the initial Fock state, is quickly lost after the net exchange of a few atoms has taken place. This process effectively measures the phase and marks the onset of a semiclassical regime in which the system undergoes Bloch oscillations around the initial particle number. These fast oscillations excite quasiparticles within each condensate and the system relaxes at a longer time scale until it displays low-energy, damped Josephson plasma oscillations, eventually coming to a halt when the equilibrium configuration is finally reached.

PACS numbers: 03.75.Fi, 05.30.Jp, 42.50.Lc, 03.65.Yz, 98.80.Bp
\end{abstract}

The way in which two Bose condensates, originally separately formed, behave when they are connected poses a fundamental problem in non-equilibrium macroscopic quantum mechanics. Two separate condensates 1 and 2 can be described by a relative number eigenstate $|n\rangle$ which in the phase representation may be written [1]

$$
|n\rangle=\int_{0}^{2 \pi} \frac{d \varphi}{\sqrt{2 \pi}} e^{i n \varphi}|\varphi\rangle,
$$

where $|\varphi\rangle$ is an eigenstate of the relative phase [2, 3] $\varphi=\varphi_{1}-\varphi_{2}$, with normalization $\left\langle\varphi \mid \varphi^{\prime}\right\rangle=\delta\left(\varphi-\varphi^{\prime}\right)$, and $n=\left(N_{1}-N_{2}\right) / 2$ is the number of transferred atoms. The Fock state (11) may be viewed as resulting from a coherent superposition of all possible values of the relative phase. This poses no problem as long as the condensates are physically separated, e.g. by a barrier so high as to be effectively impenetrable, since then no physical observable depends on $\varphi$. The situation changes when the two condensates are brought into connection, e.g. by lowering the barrier to the point where Josephson tunnelling becomes appreciable. Then a nonzero supercurrent develops which depends on the phase through the relation

$$
\dot{n}=I(\varphi)=\left(E_{J} / \hbar\right) \sin \varphi,
$$

where $E_{J}$ is the Josephson coupling energy. If we interpret the Josephson relation (2) as an operator identity, we conclude that a system initially prepared in the state (11) will display a coherent superposition of states with macroscopically different values of the Josephson current (2). After some time, these different values of the current will give rise to macroscopically different values of the relative particle number

$$
n(t)=n_{0}+\int_{0}^{t} I d t .
$$

The resulting macroscopic coherence is fragile and likely to be quickly lost by a tiny perturbation from the environment. After decoherence sets in, the relative phase is effectively measured and the system is expected to be describable by a reduced density matrix that is asymptotically diagonal in the phase representation. We will show that such a density matrix is a uniform mixture of pure state density matrices, each of them lying in the semiclassical regime where phase and number may be taken as simultaneously well-defined. In general the system finds itself very far from equilibrium and, due to the interaction with the dissipative environment, it eventually relaxes to the equilibrium configuration.

The purpose of this work is to analyze such a dynamical process. We assume that dissipation is caused by the spontaneous creation of quasiparticles within each well due to a fluctuating number of particles in the condensate that is caused in turn by the coherent exchange of atoms. Such an exchange can, of course, occur only in the presence of a physical coupling, e.g. of the Josephson type, between the two condensates. When the Josephson coupling is operating, the Hamiltonian of the system at low energies may be written [四, 可]

$$
H=E_{J}(1-\cos \varphi)+E_{c} n^{2} / 2,
$$

where $E_{c}$ represents (up to a constant) the effects of the interatomic interaction for small values of $n$. When $E_{J}$ becomes nonzero, $n$ is no longer a constant of motion, as explicitly indicated by the equation of motion (2), and its dynamic fluctuations will induce excitation of quasiparticles within each well. The description of such a process will require going beyond the pendulum Hamiltonian (4) by including the dissipative coupling to the quasiparticle field. In practice, there may be other dissipative mechanisms, such as incoherent exchange of single atoms [4] or coupling to the walls of a cavity [6], but quasiparticle creation is an intrinsic mechanism which lends itself to an approximate analytical treatment.

The problem which we address in this article is fundamental in several respects. First, it presents an im- 
portant case of (phase) quantum measurement that can be treated in detail. Second, the connection between independent BEC's has the essential features of a macroscopic interference experiment $[7,8,9,10,11,12 \pi$, since it involves an initially random relative phase which becomes well defined, in this case due to the introduction of a Josephson coupling with the help of the quasiparticle field. Finally, the establishment of phase coherence between two recently connected condensates has bearing on the "Kibble problem" of formation of cosmological singularities in the early stages of the Universe 13. Recent work on condensate coherence in optical lattices [14] suggests that the study of phase dynamics after nonadiabatic switching of the Josephson coupling is within experimental reach.

The analytical treatment which we present in this article requires the introduction of some approximations. We have already said that dissipation is assumed to be caused by the creation of intrawell quasiparticles caused by a fluctuating condensate number. Following ref. [15], we take box normalization for the one-atom wave functions within each well. This is not an important limitation in the context of laser confined alkali condensates. The initial relative particle number is assumed to be sufficiently large for the semiclassical approximation to be applicable just after decoherence takes over between distant phase values. We also assume that the two independently prepared condensates are identical and neglect quasiparticle tunneling. For conceptual simplicity, we will focus here on the case in which macroscopic quantum self-trapping takes place [16, 17]. This requires $E_{c} n_{0}^{2}>4 E_{J}$. In the future it will be desirable to extend the present analysis by relaxing some of these approximations.

The picture that emerges from our theoretical study is schematically depicted in (Fig. 1). There are at least three time scales in the connection process. First, decoherence between different phase values takes place after a few atoms have been exchanged. This process effectively measures the macroscopic phase. The second time scale is the Bloch oscillation period around the initial value of the relative particle number. These oscillations are a manifestation of the ac Josephson effect due to a chemical potential difference. Finally, the system relaxes in a longer time scale towards the equilibrium configuration. Damping is due to the irreversible energy transfer from the quickly oscillating number-phase degree of freedom to the quasiparticle field within each well.

First we perform a semiclassical study in which the relative phase and number are taken as $c$-numbers while the quasiparticle field is treated quantum mechanically. The semiclassical assumption is later justified from a full quantum treatment which explicitly shows how the quasiparticle field transforms the reduced density matrix of the phase-number coordinate from that of a pure Fock state to a mixture of Gaussian wave packets. This is the process that effectively "measures" the phase and makes it well-defined in a particular experimental realization.

At the semiclassical level (where the question of identity or not between the states $|\varphi\rangle$ and $|\varphi+2 \pi\rangle$ can be neglected), it is clear from Eq. (4), that the problem maps on to that of the the "Bloch oscillations" of a particle in a tight-binding lattice which is subject to a harmonic potential, with $n$ playing the role of (discrete) coordinate and $\varphi$ that a of a wave vector. A simple semiclassical analysis reveals that a particle placed with initial "wave vector" $\varphi_{0}$ at a high point $n_{0}$ of the harmonic tight-binding lattice, undergoes periodic motion. Specifically, for $E_{c} n_{0}^{2} \gg E_{J}$, and with an appropriate choice of time origin, we may write

$$
n(t)=\bar{n}+p \sin \left(\omega_{0} t\right),
$$

where $p \equiv\left(E_{J} / \bar{n} E_{c}\right), \omega_{0} \equiv E_{c} \bar{n} / \hbar$, and, initially, $\bar{n} \simeq n_{0}$. Equation (5) describes Bloch oscillations undergone by a quantum particle in a tilted lattice. The resulting selftrapping is not stable because the rapidly oscillating $n(t)$ induces excitations in the quasiparticle field, which acts as a dissipate environment for the macroscopic degree of freedom 18, 19. These oscillations are very fast and one is rather interested in the slower dynamics of the time average $\bar{n}$ as it experiences damping.

Since quasiparticles within each well are sensitive to the number of particles in the condensate to the extent that they are but linear deviations from the mean field description 20, it is clear that (5) yields a time-dependent perturbation that will excite quasiparticles. The timedependent Hamiltonian in e.g. the left well is 21]

$$
\begin{aligned}
& H(t)=\sum_{k}\left[\varepsilon_{k}+\frac{g}{V}\left(\frac{N}{2}+n(t)\right)\right] c_{k}^{\dagger} c_{k} \\
& +\sum_{k} \frac{g}{2 V}\left(\frac{N}{2}+n(t)\right)\left(c_{k}^{\dagger} c_{-k}^{\dagger}+c_{-k} c_{k}\right),
\end{aligned}
$$

where $g$ is the coupling constant, $N$ is the total particle number, and $V$ is the volume of one box. The Hamiltonian for the right well looks like (6) with $n(t)$ replaced by by $-n(t)$. Eq. (6) may be rewritten as

$$
H(t)=H_{0}+A p \sin (\omega t),
$$

where $H_{0}$ is the unperturbed "bath" Hamiltonian [with $n(t)$ replaced by $\bar{n}]$ and

$$
A=\frac{g}{2 V} \sum_{k}\left(2 c_{k}^{\dagger} c_{k}+c_{k}^{\dagger} c_{-k}^{\dagger}+c_{-k} c_{k}\right)
$$

By applying linear response theory [22] with $\bar{n}$ treated as constant, we obtain for the dissipated power within one well:

$$
W=\left(p^{2} / 2\right) \omega_{0} \chi^{\prime \prime}\left(\omega_{0}\right)
$$

where

$$
\chi^{\prime \prime}(\omega) \equiv \frac{1}{2 \hbar} \int d t e^{i \omega t}\langle[A(t), A(0)]\rangle
$$




$$
\begin{aligned}
& =\frac{\pi g^{2}}{2 \hbar V^{2}} \sum_{k}\left|u_{k}-v_{k}\right|^{4} \operatorname{coth}\left(\frac{\hbar \omega_{k}}{2 k_{B} T}\right) \delta\left(\omega-2 \omega_{k}\right) \\
& \simeq \frac{\hbar V}{128 \pi N^{2} c^{3}} \omega^{4} \operatorname{coth}\left(\frac{\hbar \omega}{4 k_{B} T}\right) .
\end{aligned}
$$

In the third line, we have taken the low frequency approximation $\hbar \omega \ll m c^{2}, c$ being the sound velocity and $m$ the atom mass [21]. Here $u_{k}$ and $v_{k}$ are the coherence factors of the Bogoliubov transformation, so that, in this limit [21], $u_{k}-v_{k}=(\hbar V c k / N g)^{1 / 2}$, where $N / 2 V$ is the atom density in one well. We note that $\chi^{\prime \prime}(\omega)$ goes like $\omega^{4}$ at low temperatures and like $\omega^{3}$ at high temperatures. The second line of Eq. (11) indicates that the quasiparticles are excited in pairs, which can be ascribed to the pairing structure of the depletion cloud [3, 23].

The energy that is dissipated into the quasiparticle field has to be taken from the energy of the macroscopic pendulum (1) which, under the self-trapping assumption, is dominated by the interaction term. Thus, we write

$$
2 W=-E_{c} \bar{n} \dot{\bar{n}},
$$

where the factor of 2 accounts for the presence of two wells. We derive the differential equation

$$
\dot{\bar{n}}=-C \bar{n}^{2} \operatorname{coth}\left(\frac{E_{c} \bar{n}}{4 k_{B} T}\right),
$$

where $C \equiv 2 \pi \eta E_{J}^{2} / \hbar N^{4} E_{c}$, with $E_{c}=2 g / V \equiv$ $8 \pi \hbar^{2} a / m V$ and $\eta \equiv\left(N a^{3} / 2 \pi V\right)^{1 / 2}$ the small parameter of the dilute gas. At low temperatures $\left(k_{B} T \ll E_{c} \bar{n}\right)$ we get

$$
\bar{n}(t)=\frac{n_{0}}{1+\gamma_{r} t},
$$

while, at high temperatures $\left(k_{B} T \gg E_{c} \bar{n}\right)$,

$$
\bar{n}(t)=n_{0} \exp \left(-\gamma_{r} t\right) .
$$

In (14) and (15), $\gamma_{r}$ is, respectively, the low and high temperature limit of the general expression

$$
\gamma_{r}=\frac{\hbar C}{E_{c}} \omega_{0} \operatorname{coth}\left(\frac{\hbar \omega_{0}}{4 k_{B} T}\right)
$$

where $\omega_{0}$ refers to $n_{0}$.

As the dissipation process continues, the energy of the macroscopic pendulum decreases until it eventually undergoes low energy harmonic oscillations. In this limit, the analysis of the dissipation process is similar to that described above, with $\omega_{0}$ replaced by the Josephson plasma frequency $\omega_{\mathrm{JP}}=\sqrt{E_{J} E_{c}} / \hbar$ and with the pendulum energy written as $\tilde{n}^{2} E_{c} / 2$, where $\tilde{n}$ is the amplitude of the number oscillations. Since $\omega_{\mathrm{JP}}$ does not depend on $\tilde{n}$ for $\tilde{n} \ll N$, we obtain for both low and high temperatures

$$
\tilde{n}(t)=\tilde{n}(0) \exp \left(-\gamma_{r} t\right)
$$

$\gamma_{r}$ being given by 16 with $\omega_{0}$ replaced by $\omega_{\mathrm{JP}}$.

It remains to justify the semiclassical approximation. For that we have performed a full quantum calculation of the phase dynamics right after the connection has been established. The direct reversal process $n \rightarrow-n$ may be safely neglected. We generalize quantum dissipation techniques [24] to the case of quadratic coupling in the bath coordinates [see Eq. (9)]. After some lengthy algebra, we find that, in the phase representation, the reduced density matrix simply evolves as

$$
\left|\rho\left(\varphi, \varphi^{\prime} ; t\right)\right|=\frac{1}{2 \pi} \exp \left[-4 \gamma_{d} t \sin ^{2}\left(\frac{\varphi-\varphi^{\prime}}{2}\right)\right],
$$

where

$$
\gamma_{d}=\gamma_{r} n_{0} \operatorname{coth}\left(\frac{E_{c} n_{0}}{2 k_{B} T},\right) .
$$

For $\gamma_{d} t \gg 1$, Eq. (18) becomes effectively equivalent to

$$
\left|\rho\left(\varphi, \varphi^{\prime} ; t\right)\right|=(2 \pi)^{-1} \exp \left[-\left(\varphi-\varphi^{\prime}\right)^{2} \gamma_{d} t\right],
$$

and hence $\gamma_{d}$ is identified as the decoherence rate. It is possible to prove that the reduced density matrix (20) is a mixture of pure state Gaussians $\sim \exp \left[-2(\varphi-\theta)^{2} \gamma_{d} t\right]$ with $\theta$ uniformly distributed between 0 and $2 \pi$. Thus we assert that the density matrix (20) describes a quantum system in which the variable $\varphi$ is measured with precision $\sim\left(\gamma_{d} t\right)^{-1 / 2}$ with the average result chosen among a menu of uniformly distributed possible values. Here the role of the "measurement apparatus" is played by the quasiparticle field with its many degrees of freedom.

At low temperatures, we obtain $\gamma_{d} / \gamma_{r}=n_{0}$. Now we note that $2 \gamma_{r}$ is the relaxation of the total energy $E_{c} n_{0}^{2} / 2$ and that $E_{c} n_{0}$ is the variation in energy due to the exchange of a single atom. We conclude that the ratio between the decoherence and the energy relaxation rates is the ratio between the total energy and the energy variation associated to the transfer of one atom. Thus decoherence is achieved as soon as $|\bar{n}(t)|$ decreases by a few units [25]. Once this has happened, Eq. (20) applies and the system may be treated semiclassically with an initial value of the phase that is chosen at random and which is defined with increasing precision as time evolves 12, 26. In other words, the decoherence rate can be identified with the rate of net atom exchange. On the contrary, an appreciable energy relaxation requires the exchange of many atoms. This picture is consistent with the idea that the net exchange of a single atom plays the role of the excitation of one quantum of the dissipative bath 27].

Once we can describe our system as a mixture of semiclassical wave packets, linear evolution applies to these even in the presence of dissipation. Since the translational motion of a Gaussian wave packet is semiclassical, we apply the method initially described with the confidence that it has been justified by a full quantum treatment. 
The final picture of the connection process is one which involves several time scales. Decoherence in the phase representation sets in after the exchange of a few atoms has been completed. For a given experimental run this can be interpreted as the measurement of the phase in which a given value is chosen with an accuracy that improves with time. After the phase is defined, a semiclassical treatment suffices to conclude that the macroscopic degree of freedom will undergo very fast Bloch oscillations that will dissipate energy into the many degrees of freedom of the quasiparticle field. Ultimately this Bloch oscillations become low energy Josephson plasma oscillations that are also damped by the quasiparticle field. At long times we expect that the system density matrix will be the thermal equilibrium one which corresponds to a total energy of $E_{c} n_{0}^{2} / 2$. For large $V$ the corresponding temperature is much smaller than the critical temperature.

The main message is that the depletion cloud, described by the quasiparticle field, can be an intrinsic agent for the decoherence and measurement of the macroscopic phase. We have studied the most fundamental intrinsic mechanism. Incoherent exchange of thermal atoms [4] and coupling to the walls of a cavity [6] will yield additional contributions to both the decoherence and the energy relaxation rates.

This work has been supported by NSF Grant no. DMR-99-86199, by the Dirección General de Investigación Científica y Técnica under Grants No. PB96-0080C02 and BFM2001-0172, and by the Ramón Areces Foundation. One of us (I.Z.) acknowledges support from the Fulbright Commission and Spain's Ministerio de Educación y Ciencia.

* Present address: Neworldcity, C/ Almagro 2, E-28010 Madrid, Spain

[1] One may allow for a classical uncertainty in the knowledge of the initial relative particle number by describing the system by an incoherent mixture of Fock states, $\rho=\sum_{n}|n\rangle \rho_{n n}\langle n|$.

[2] We will neglect throughout this paper effects which tend to zero in the limit $N \rightarrow \infty$, cf. Ref. [3], sec. VII.C.

[3] A. J. Leggett, Rev. Mod. Phys. 73, 307 (2001).

[4] I. Zapata, F. Sols, and A. J. Leggett, Phys. Rev. A 57, R28 (1998).

[5] F. Sols, in Bose-Einstein Condensation in Atomic Gases, M. Inguscio, S. Stringari, and C. E. Wieman, eds. (IOS Press, Amsterdam, 1999).

[6] D. Jaksch et al. Phys. Rev. Lett. 86, 4733 (2001).

[7] M. R. Andrews et al., Science 275, 637 (1997).

[8] B. P. Anderson and M. A. Kasevich, Science 282, 1686 (1998).

[9] P. W. Anderson, Basic Notions of Condensed Matter Physics (Benjamin, Menlo Park, 1984).
[10] A. J. Leggett and F. Sols, Found. Phys. 21, 353 (1991).

[11] Y. Castin and J. Dalibard, Phys. Rev. A 55, 4330 (1997).

[12] S. Kohler and F. Sols, Phys. Rev. A 63, 053605 (2001).

[13] T. W. B. Kibble, J. Phys. A 9, 1387 (1976); W. H. Zurek, Nature 317, 505 (1985).

[14] M. Greiner et al., Nature 415, 39 (2002).

[15] F. Meier and W. Zwerger, Phys. Rev. A 64, 033610 (2001).

[16] A. Smerzi et al., Phys. Rev. Lett. 79, 4950 (1997).

[17] G. J. Milburn et al., Phys. Rev. A 55, 4318 (1997).

[18] J. Ruostekoski and D. F. Walls, Phys. Rev. A 58, R50 (1998).

[19] A. J. Leggett and S. Takagi, Ann. Phys. (New York) 106, 79 (1977).

[20] F. Dalfovo, S. Giorgini, L. P. Pitaevskii, and S. Stringari, Rev. Mod. Phys. 71, 463 (1999).

[21] A. L. Fetter, Quantum Many Particle Systems (McGrawHill, New York, 1971).

[22] D. Forster, Hydrodynamic Fluctuations, Broken Symmetry, and Correlation Functions (Benjamin, Menlo Park 1975).

[23] A. L. Fetter, Ann. Phys. (New York) 70, 67 (1972).

[24] U. Weiss, Quantum Dissipative Systems (World Scientific, Singapore, 1999).

[25] This suggests that, in principle, a fine measurement of $n$ at different times in various runs would reveal $\gamma_{d}$ if $n_{0}$ is known precisely.

[26] Eventually the phase spread $\sim 1 / \sqrt{\gamma_{d} t}$ will saturate to a finite value due to interactions, which cause quantum fluctuations of the phase that tend to oppose definition in a given experimental run [12]. We neglect this effect here.

[27] A. O. Caldeira and A. J. Leggett, Phys. Rev. A 31, 1059 (1985); W. G. Unruh and W. H. Zurek, Phys. Rev. D 40 , 1071 (1989).

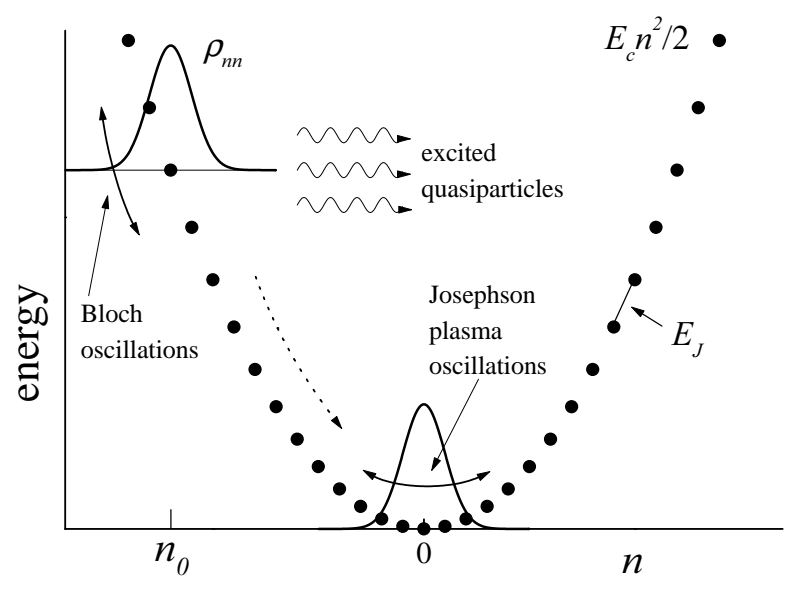

FIG. 1: Schematics of the connection process in the number representation: After a time $t \sim 1 / \gamma_{d}$ a semiclassical wave packet forms that undergoes Bloch oscillations thus exciting quasiparticles. For $t \gtrsim 1 / \gamma_{r}$, the system relaxes its energy, eventually performing underdamped Josephson plasma oscillations. 